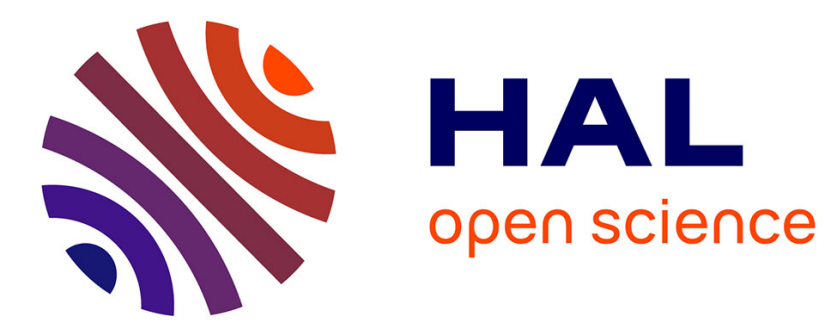

\title{
Sequential Monte Carlo for rare event estimation
}

Frédéric Cérou, Pierre del Moral, Teddy Furon, Arnaud Guyader

\section{To cite this version:}

Frédéric Cérou, Pierre del Moral, Teddy Furon, Arnaud Guyader. Sequential Monte Carlo for rare event estimation. Statistics and Computing, 2012, 22 (3), pp.795-908. 10.1007/s11222-011-9231-6 . inria-00584352

\section{HAL Id: inria-00584352 \\ https://hal.inria.fr/inria-00584352}

Submitted on 26 Sep 2013

HAL is a multi-disciplinary open access archive for the deposit and dissemination of scientific research documents, whether they are published or not. The documents may come from teaching and research institutions in France or abroad, or from public or private research centers.
L'archive ouverte pluridisciplinaire HAL, est destinée au dépôt et à la diffusion de documents scientifiques de niveau recherche, publiés ou non, émanant des établissements d'enseignement et de recherche français ou étrangers, des laboratoires publics ou privés. 


\title{
SEQUENTIAL MONTE CARLO FOR RARE EVENT ESTIMATION*
}

\author{
By Frédéric CÉrou ${ }^{\ddagger}$, Pierre Del Moral ${ }^{\dagger, \S}$, \\ TEDdy Furon ${ }^{\ddagger}$, AND ARNAUd GUYADER ${ }^{\ddagger}, \boldsymbol{q}$ \\ INRIA Rennes - Bretagne Atlantique $e^{\ddagger}$ INRIA Bordeaux Sud-Ouest ${ }^{\S}$ and \\ Université de Haute Bretagne
}

\begin{abstract}
This paper discusses a novel strategy for simulating rare events and an associated Monte Carlo estimation of tail probabilities. Our method uses a system of interacting particles and exploits a FeynmanKac representation of that system to analyze their fluctuations. Our precise analysis of the variance of a standard multilevel splitting algorithm reveals an opportunity for improvement. This leads to a novel method that relies on adaptive levels and produces estimates with optimal variance.

The motivation for this theoretical work comes from problems occurring in watermarking and fingerprinting of digital contents, which represents a new field of applications of rare event simulation techniques. Some numerical results show the performance of our technique for these practical applications.
\end{abstract}

1. Introduction. Monte Carlo approach is a common tool to estimate the expectation of any function of a random object when analytical or numerical methods are not available. However, if the function is non-zero on a set which has a very small probability, then the naive Monte Carlo method will return the estimate zero, unless the sample size is so large that it becomes intractable. Typically we want to estimate precisely and in a reasonable time the small probability, say $10^{-9}$ or below, of an extreme event. A naive Monte Carlo method is impractical as it would require an excessively large sample. To circumvent this difficulty, importance sampling (see e.g. Bucklew [4]) changes the law of the simulated random objects, and reweighs the sample consequently. The difficulty is then to choose the appropriate change of probability in order to achieve a good estimate. This is not always obvious, especially when there is no large deviation result to consider.

Importance splitting, or multilevel splitting, is another approach that is well

${ }^{*}$ This work was partially supported by the French Agence Nationale de la Recherche (ANR), project Nebbiano, number ANR-06-SETI-009.

${ }^{\dagger}$ Corresponding author. E-mail: Pierre.Del_Moral@inria.fr

AMS 2000 subject classifications: Primary 65C05; secondary 65C35, 60F05, 62G30

Keywords and phrases: Sequential Monte Carlo Methods, Rare Event Analysis 
adapted when the random object is a Markovian process. The basic idea is to reinforce trajectories that approach the targeted set by splitting (or branching) them and discarding the others. This very powerful approach in fact dates back to Kahn and Harris [17] and Rosenbluth and Rosenbluth [23]. We refer the reader to the paper by Glasserman et al. [14] which contains a precise review on these methods as well as a detailed list of references. Recently, the connection between importance splitting for Markovian processes and particle methods for Feynman-Kac models has lead to some improvements and to a rigorous framework for mathematical analysis (see Del Moral [9]). Unlike previous works concerning rare event estimation and simulation, the present work deals with rare events for a fixed probability distribution. We are simply concerned here with events of the type $\{X \in A\}$ for some random vector $X$, with $p=\mathbb{P}(X \in A)=\mathbb{P}(\Phi(X)>L) \ll 1$, where $\Phi$ is a mapping from $\mathbb{R}^{d}$ to $\mathbb{R}$, and where there is no dynamical model for $X$, i.e. $X$ is not a process indexed by the time. In order to use the framework developed for Markov processes (see Cérou et al. [6], Cérou and Guyader [7], Del Moral and Lezaud [11]), we construct a family of Markov transition kernels $M_{k}$ whose invariant measures are the successive laws of $X$ restricted on smaller and smaller sets, the smallest being $A$. As usual when using a splitting technique in rare event simulation, we decompose the rare event in not so rare nested events, whose product of probabilities equals the probability of the rare event.

Del Moral et al. [10] and Johansen et al. [16] were first to use fixed-levels algorithms for static rare events. These articles were written in a different framework, and thus do not deal with the practical details of our precise setting. In the present article, we detail both a fixed and an adaptive multilevels algorithm, the adaptive one consisting in optimally placing the levels on the fly.

Recently and independently from the authors of the present work, Botev and Kroese [3] proposed the same approach. These authors work on a similar algorithm, including the use of quantiles of the random variable $\Phi(X)$ on the swarm of particles in order to estimate the next level. The main difference is their two stage procedure (like in Garvels [13]): they first run the algorithm just to compute the levels, and then they restart from the beginning with these proposed levels. Actually we prove that by computing the levels on the fly (within the same run as the one to compute the rare event probability), we only pay a small bias on the estimate. Note also that [3] does not address the general construction of the transition kernels $M_{k}$, since the authors only tackle examples where they can derive a Gibbs sampler at each step. This is mainly possible because their function $\Phi$ is linear, which 
is a severe restriction.

Another related approach is the recent work on combinatorial counting of Rubinstein [24]. This article deals with optimization and counting problems in which $X$ has a uniform distribution over a discrete but very large state space. The author uses what he calls a cloning procedure, where the number of offsprings is fixed (i.e. the same for all the particles in the sample) but adaptive to keep the number roughly constant, while removing redundant particles after the MCMC step. This is a main difference since we use a resampling with replacement procedure. Finally, we would like to mention that these last two papers [3][24] have demonstrated the performance of their algorithms via an extensive simulation study, to which we now lay out the mathematical foundations.

The paper is organized as follows. Section 2 describes and analyses the fixedlevels algorithm. Section 3 provides and studies the adaptive levels version, which proves to be optimal in terms of asymptotic variance of the estimator. Section 4 deals with the tuning of the algorithm and especially the choice and the iteration of the transition kernel which is at the core of the method. Section 5 shows the relevance of our algorithm for watermarking and fingerprinting, which constitute a new application area of rare event simulation techniques. Finally, all the proofs are gathered in the appendix.

\section{The fixed-levels method.}

2.1. Assumptions and ingredients. We assume that $X$ is a random element on $\mathbb{R}^{d}$ for some $d>0$, and denote by $\mu$ its probability distribution on the underlying probability space $(\Omega, \mathcal{F}, \mathbb{P})$. We denote by $A$ the rare set of interest, and we assume that $A=\left\{x \in \mathbb{R}^{d}\right.$ s.t. $\left.\Phi(x) \geq L\right\}$ for some function $\Phi: \mathbb{R}^{d} \mapsto \mathbb{R}$ and some real number $L$. We also assume that we know how to draw i.i.d. samples from $\mu$.

Our algorithm makes use of the following ingredients. An increasing sequence $\left\{L_{0}, \ldots, L_{n}\right\}$ in $\overline{\mathbb{R}}$, with $L_{0}=-\infty$ and $L_{n}=L$ defines a sequence of corresponding sets $A_{k}=\left\{x \in \mathbb{R}^{d}, \Phi(x) \geq L_{k}\right\}$. These sets are thus nested: $\mathbb{R}^{d}=A_{0} \supset A_{1} \supset \cdots \supset A_{n}=A$. We now need to choose the sequence $\left\{L_{0}, \ldots, L_{n}\right\}$ in such a way that $p_{k}=\mathbb{P}\left(X \in A_{k} \mid X \in A_{k-1}\right)$ is not too small. For indices $k>n$, we assume that $L_{k}=L_{n}$ and $A_{k}=A_{n}$. We also need to choose a Markov transition kernel $K$ on $\mathbb{R}^{d}$ which is $\mu$-symmetric, that is

$$
\forall(x, y) \in \mathbb{R}^{d} \times \mathbb{R}^{d}, \mu(d x) K(x, d y)=\mu(d y) K(y, d x) .
$$

As a consequence, $K$ has $\mu$ as an invariant measure.

As we will see in the sequel, the choice of the $L_{k}$ 's can be made adaptive and is thus not an issue. However, the choice of the kernel $K$ is crucial. Even 
if any $\mu$-symmetric kernel would eventually do the job, we need to carefully choose it to make the algorithm efficient as discussed in section 4.

Consider now a Markov chain $\left(X_{k}\right)_{k \geq 0}$ defined by: $\mathcal{L}\left(X_{0}\right)=\mu$ and the inhomogeneous transitions kernels $\mathbb{P}\left(X_{k} \in d y \mid X_{k-1}=x\right)=M_{k}(x, d y)$, with

$$
M_{k}(x, d y)=\mathbb{1}_{A_{k}^{c}}(x) \delta_{x}(d y)+\mathbb{1}_{A_{k}}(x)\left(K(x, d y) \mathbb{1}_{A_{k}}(y)+K\left(x, A_{k}^{c}\right) \delta_{x}(d y)\right) .
$$

Moving a particle according to $M_{k}$ is then twofold: firstly a new transition according to $K$ is proposed, and secondly we accept this transition only if it stays in $A_{k}$, keeping the old position otherwise. For $k \in\{0, \ldots, n\}$, denote

$\mu_{k}(d x)=\frac{1}{\mu\left(A_{k}\right)} \mathbb{1}_{A_{k}}(x) \mu(d x)$ the normalized restriction of $\mu$ on $A_{k}$. The following stationarity property holds for $\mu$ and $\mu_{k}$.

Proposition 1. The measures $\mu$ and $\mu_{k}$ are both invariant by the transition kernel $M_{k}$.

From a general point of view, a Feynman-Kac representation for $\mu_{k}$ is a formula of the form

$$
\mu_{k}(\varphi)=\frac{\mathbb{E}\left[\varphi\left(X_{k}\right) \prod_{m=0}^{k-1} G_{m}\left(X_{m}\right)\right]}{\mathbb{E}\left[\prod_{m=0}^{k-1} G_{m}\left(X_{m}\right)\right]},
$$

where the potentials $G_{m}$ are positive functions, and $\left(X_{k}\right)_{k \geq 0}$ is a non homogeneous Markov chain with transitions $M_{k}$. If we know how to draw realizations of the Markov chain, then we can compute $\mu_{k}(\varphi)$ with a Monte Carlo approach. But naive Monte Carlo is not efficient, because most of the realizations of the chain have small values for the product of the potentials. Anyway, in this form a much nicer Monte Carlo algorithm can be used. It mainly consists in keeping a cloud of particles $\left(\xi_{k}^{j}\right)$, with time $0 \leq k \leq n$ and particle index $1 \leq j \leq N$. Then for each time step $k$, discard those with small potential $G_{k}$, and branch the others, with a rate proportional to $G_{k}\left(\xi_{k}^{j}\right)$. Then apply the Markov transition $M_{k}$ to all the surviving particles, and iterate on the time step.

This approach has given birth to a huge amount of literature, and is often referred to as Sequential Importance Sampling (SIS) or Sequential Monte Carlo (SMC). See the monograph by Del Moral [9] for a theoretical overview and Doucet et al. [12] for examples of applications. In our context, the Feynman-Kac representation for $\mu_{k}$ has the following form.

Proposition 2. For every test function $\varphi$, for $k \in\{0, \ldots, n\}$, the FeynmanKac representation is as follows

$$
\begin{aligned}
& \qquad \mu_{k}(\varphi)=\frac{\mathbb{E}\left[\varphi\left(X_{k}\right) \prod_{m=0}^{k-1} \mathbb{1}_{A_{m+1}}\left(X_{m}\right)\right]}{\mathbb{E}\left[\prod_{m=0}^{k-1} \mathbb{1}_{A_{m+1}}\left(X_{m}\right)\right]} \\
& \text { imsart-aos ver. } 2010 / 04 / 27 \text { file: cdfg.tex date: July 2, } 2010
\end{aligned}
$$


where $\left(X_{k}\right)_{k \geq 0}$ is a Markov chain given by the following conditions: $X_{0} \sim \mu$ and the inhomogeneous transition kernels $\left(M_{k}\right)_{k \geq 1}$.

2.2. The fixed-levels algorithm. Proposition 2 shows that the framework of Feynman-Kac formulae does apply, and thus this grants access to the approximation of the associated measures using an interacting particle method as studied by Del Moral in [9]. Basically, at each iteration $k$, it consists in selecting the particles according to the potentials, here $\mathbb{1}_{A_{k+1}}$, and then in propagating the particles according to the transitions given by $M_{k+1}$.

The approximation of the rare event probability stems from the following obvious property

$$
p=\mathbb{P}\left(X \in A_{n}\right)=\prod_{k=0}^{n-1} \mathbb{P}\left(X \in A_{k+1} \mid X \in A_{k}\right)=\prod_{k=0}^{n-1} \mu_{k}\left(A_{k+1}\right)
$$

and finally

$$
p=\prod_{k=0}^{n-1} \frac{\mathbb{E}\left[\mathbb{1}_{A_{k+1}}\left(X_{k}\right) \prod_{m=0}^{k-1} \mathbb{1}_{A_{m+1}}\left(X_{m}\right)\right]}{\mathbb{E}\left[\prod_{m=0}^{k-1} \mathbb{1}_{A_{m+1}}\left(X_{m}\right)\right]},
$$

where the last equality comes from Proposition 2. We approximate at each stage $p_{k}=\mathbb{P}\left(X \in A_{k+1} \mid X \in A_{k}\right)$ by the proportion of the particles already in the next set, and the total probability is estimated as the product of those. This gives the algorithm 1.

\section{Algorithm 1.}

\section{Parameters}

$N$ the number of particles, the sequence $\left\{L_{0}, \ldots, L_{n}\right\}$ of levels.

\section{Initialization}

Draw an i.i.d. $N$-sample $\left(\xi_{0}^{j}\right)_{1 \leq j \leq N}$, of the law $\mu$.

\section{Iterations}

for $k=0$ to $n-1 / *$ level number */

Let $I_{k}=\left\{j: \xi_{k}^{j} \in A_{k+1}\right\}$.

Let $\hat{p}_{k}=\frac{\left|I_{k}\right|}{N}$.

for $j=1$ to $N / *$ particle index */

Let $\tilde{\xi}_{k+1}^{j}$ be a copy of $\xi_{k}^{\ell}$ where $\ell$ is chosen randomly in $I_{k}$ with uniform probabilities.

Draw a new particle $\hat{\xi}_{k+1}^{j} \sim K\left(\tilde{\xi}_{k+1}^{j},.\right)$.

imsart-aos ver. 2010/04/27 file: cdfg.tex date: July 2, 2010 
If $\hat{\xi}_{k+1}^{j} \in A_{k+1}$ then let $\xi_{k+1}^{j}=\hat{\xi}_{k+1}^{j}$, and $\xi_{k+1}^{j}=\tilde{\xi}_{k+1}^{j}$ otherwise.

endfor

endfor

\section{Output}

Estimate the probability of the rare event by $\hat{p}=\prod_{k=0}^{n-1} \hat{p}_{k}$.

The last set of particles is a (non independent) sample that provides a good approximation of the law $\mu_{n}$ of the rare event.

2.3. Fluctuations analysis. Del Moral has extensively studied in a very general context the asymptotic behavior of the interacting particle model as the number $N$ of particles goes to infinity [9]. For example, it is well known that the estimate $\hat{p}$ is unbiased. The next proposition presents a precise fluctuation result in our context of rare event analysis.

Proposition 3. Let $\hat{p}$ denote the estimate given by the fixed-levels algorithm, then

$$
\sqrt{N} \frac{\hat{p}-p}{p} \underset{N \rightarrow+\infty}{\stackrel{\mathcal{D}}{\longrightarrow}} \mathcal{N}\left(0, \sigma^{2}\right)
$$

with

$$
\begin{aligned}
\sigma^{2}= & \sum_{k=0}^{n-1} \frac{1-p_{k}}{p_{k}}+ \\
& \sum_{k=0}^{n-1} \frac{1}{p_{k}} \mathbb{E}\left[\left(\frac{\mathbb{P}\left(X_{n-1} \in A_{n} \mid X_{k}\right)}{\mathbb{P}\left(X_{n-1} \in A_{n} \mid X_{k-1} \in A_{k}\right)}-1\right)^{2} \mid X_{k-1} \in A_{k}\right] .
\end{aligned}
$$

The samples are not independent due to the splitting of successful particles. In fact the variance is lower bounded

$$
\sigma^{2} \geq \sum_{k=0}^{n-1} \frac{1-p_{k}}{p_{k}}
$$

with equality if and only if for all $k=0, \ldots, n-1$ and knowing that $X_{k-1} \in$ $A_{k}$, one has

$$
\mathbb{P}\left(X_{n-1} \in A_{n} \mid X_{k}\right) \perp X_{k} .
$$

This means that equality holds if, between step $k$ and step $n-1$, the algorithm forgets the initial position $X_{k}$. In order to reach this goal, a possible 
route is to begin step $k$ by applying an infinite number of times (and not only one time as is the case in Algorithm 1) the transition kernel $M_{k}$ with stationary distribution $\mu_{k}=\mathcal{L}\left(X \mid X \in A_{k}\right)$. We will discuss this point in details in section 4 .

Anyway, from now on, we assume that at each step $k$ it is possible to draw an i.i.d. sample of the law of $X$ conditionally on the event $\left\{X \in A_{k}\right\}=$ $\left\{\Phi(X)>L_{k}\right\}$. Then the relative variance of the estimator reduces to:

$$
\sigma^{2}=\sum_{k=0}^{n-1} \frac{1-p_{k}}{p_{k}}
$$

Thus, for a fixed value of $p$ and a fixed number $n$ of levels, this asymptotic variance would be minimal if $p_{k} \equiv p_{0}$ for all $k$. This is indeed a simple constrained optimization problem:

$$
\arg \min _{p_{0}, \ldots, p_{n-1}} \sum_{k=0}^{n-1} \frac{1-p_{k}}{p_{k}} \quad \text { s.t. } \quad \prod_{k=0}^{n-1} p_{k}=p .
$$

In this case, the minimal asymptotic variance is simply $n \frac{1-p_{0}}{p_{0}}$, with $p_{0}=p^{\frac{1}{n}}$. This optimal situation corresponds to the case where the levels are evenly spaced in terms of probability of success: as far as multilevel splitting for Markov processes is concerned, this point was also mentioned in Glasserman et al. [14], Lagnoux [18] and Cérou et al. [6]. The following section addresses this crucial issue for the adaptive version of the algorithm. Before this, this section ends with two remarks.

\section{Remarks:}

1. If one's particular interest is the variance of $\hat{p}$ rather than a convergence in distribution like the CLT-type result of Proposition 3, then we can turn to the recent non asymptotic results obtained in Cérou et al. [5, corollary 5.2]. Under some regularity conditions (mainly about the mixing property of the kernel $K$ ), there exist positive constants $\alpha_{k}$, for $0 \leq k \leq n-1$, such that for all $N \geq N_{0}=\sum_{k=0}^{n-1} \frac{\alpha_{k}}{p_{k}}$,

$$
\mathbb{E}\left(\left[\frac{\hat{p}-p}{p}\right]^{2}\right) \leq 4 \frac{N_{0}}{N}
$$

If we assume an i.i.d. sample, then all the $\alpha_{k}$ 's are all equal to 1 , and $N_{0}=\sum_{k=0}^{n-1} \frac{1}{p_{k}}$. 
2. Finally, there is a maybe small, but non-zero, probability that the particle system dies at some stage. This may typically happen when two consecutive levels are too far apart, or when the number of particles is too small. A first solution to this problem is given in Le Gland and Oudjane [19]. The idea is to go on sampling new particles until a given number of them have reached the next level. The price to pay is a possibly very long computation time. A second solution is proposed in the next section.

\section{The adaptive method.}

3.1. The algorithm. As we may not have a great insight about the law $\mu$ and/or the mapping $\Phi$, typically when $\Phi$ is a 'black box', the choice of the levels $L_{1}, \ldots, L_{n-1}$ might prove to be quite problematic. We propose from now on to adaptively choose the levels, ensuring not only that the particle system never dies but also that the asymptotic variance of the estimate $\hat{p}$ is minimized.

The method is indeed simple to implement. We choose a prescribed success rate $p_{0}$ between two consecutive levels $\left(e . g . p_{0}=0.75\right)$. After application of the kernel $M_{k}$, the algorithm sorts the particles $\xi_{k}^{j}$ according to their scores $\Phi\left(\xi_{k}^{j}\right)$. Then it sets the next level to the $\left(1-p_{0}\right)$ empirical quantile $\hat{L}_{k+1}$, which means that a proportion $p_{0}$ of the particles scores are above it. Starting from this sample of $p_{0} N$ particles which are independently and identically distributed according to the law $\mathcal{L}\left(X \mid \Phi(X)>\hat{L}_{k+1}\right)$, an i.i.d. sample of size $N$ is drawn with the same distribution, and the rest of the algorithm is unchanged.

The algorithm then stops when some $\hat{L}_{\hat{n}_{0}+1} \geq L$, and the probability is estimated by $\hat{p}=\hat{r}_{0} p_{0}^{\hat{n}_{0}}$, where $\hat{r}_{0}$ denotes the number of particles in the last iteration being above level $L$. The number $\hat{n}_{0}$ of steps is random, but if $N$ is large enough, then Lemma 1 in Appendix proves that, outside an event of exponentially small probability, $\hat{n}_{0}$ is actually fixed by the ratio of the logarithms

$$
n_{0}=\left\lfloor\frac{\log \mathbb{P}(X \in A)}{\log p_{0}}\right\rfloor=\left\lfloor\frac{\log p}{\log p_{0}}\right\rfloor .
$$

As mentioned above, this variant enforces evenly spaced levels in terms of probability of success, and therefore a minimal asymptotic variance for the estimate $\hat{p}$ of $p$. The pseudo-code for the adaptive algorithm is given in Algorithm 2 below.

imsart-aos ver. 2010/04/27 file: cdfg.tex date: July 2, 2010 
Algorithm 2.

\section{Parameters}

$N$ the number of particles, the number $N_{0}<N$ of succeeding particles, and let $p_{0}=N_{0} / N$.

\section{Initialization}

Draw an i.i.d. $N$-sample $\left(\xi_{0}^{j}\right)_{1 \leq j \leq N}$ of the law $\mu$.

Compute $\hat{L}_{1}$, the $\left(1-p_{0}\right)$ quantile of $\Phi\left(\xi_{0}^{j}\right), j=1, \ldots, N$. $k=1$;

\section{Iterations}

while $\hat{L}_{k}<L$ do

Starting from an i.i.d. $p_{0} N$-sample with law $\mathcal{L}\left(X \mid \Phi(X)>\hat{L}_{k}\right)$, draw an i.i.d. $N$-sample $\left(\xi_{k}^{j}\right)_{1 \leq j \leq N}$ with the same law.

Compute $\hat{L}_{k+1}$, the $\left(1-p_{0}\right)$ quantile of $\Phi\left(\xi_{k}^{j}\right), j=1, \ldots, N$. $k=k+1$;

endwhile

Let $N_{L}$ the number of particles $\xi_{k-1}^{j}, j=1, \ldots, N$, such that $\Phi\left(\xi_{k-1}^{j}\right) \geq$ $L$.

\section{Output}

Estimate the probability of the rare event by $\hat{p}=\frac{N_{L}}{N} p_{0}^{k-1}$.

The last set of particles is a (non independent) sample that provides a good approximation of the law $\mu_{n}$ of the rare event. 


\section{Remarks:}

1. In this algorithm, the step drawing an $N$-sample starting from a $p_{0} N$ sample is of course the trickiest one. The analytical study of the next subsection assumes it can be done perfectly. In section 4 , we propose a way to implement it in practice, at least approximately, and section 5.1 shows its practical efficiency.

2. The costs of adaptive levels is a higher complexity by a factor $\log N$ (due to the quick sort), and a slight loss of accuracy due to a bias. Yet, Proposition 4 proves that this bias becomes negligible compared to the standard deviation as $N$ increases. Experimental results of section 5.1 illustrate this.

3. Estimation of quantiles: Some applications require the estimates of quantiles of the random variable $\Phi(X)$. This can be done at no additional cost within the previous algorithm. For $\alpha \in(0,1)$, define the $\alpha$-quantile by $q_{\alpha}=\sup \{x: P(\Phi(X) \leq x) \leq \alpha\}$. When the algorithm is in step $k$, with a set of particles $\left\{\xi_{k}^{j}, j=1, \ldots, N\right\}$, such that $p_{0}^{k+1} \leq$ $1-\alpha<p_{0}^{k}$, then let $r=1-(1-\alpha) p^{-k}$. An estimate of the quantile $q_{\alpha}$ is then given by the $r$ quantile of the sample $\left\{\Phi\left(\xi_{k}^{j}\right), j=1, \ldots, N\right\}$.

3.2. Bias and variance. The assumption of a continuous cumulative distribution function (cdf) $F$ of $\Phi(X)$ is now required to derive the properties of the adaptive algorithm. Let us write the rare event probability as

$$
p=r_{0} p_{0}^{n_{0}}, \text { with } n_{0}=\left\lfloor\frac{\log p}{\log p_{0}}\right\rfloor \text { and } r_{0}=p p_{0}^{-n_{0}}
$$

so that $r_{0} \in\left(p_{0}, 1\right]$. In the same way we write $\hat{p}=\hat{r}_{0} p_{0}^{\hat{n}_{0}}$, with $\hat{n}_{0}$ the number of steps before the algorithm stops. A first theorem shows a CLTtype convergence.

THEOREM 3.1. If $F$ is continuous, then we have

$$
\sqrt{N}(\hat{p}-p) \underset{N \rightarrow+\infty}{\stackrel{\mathcal{D}}{\longrightarrow}} \mathcal{N}\left(0, \sigma^{2}\right)
$$

where

$$
\sigma^{2}=p^{2}\left(n_{0} \frac{1-p_{0}}{p_{0}}+\frac{1-r_{0}}{r_{0}}\right) .
$$

Unlike the fixed-levels version of the algorithm, the adaptive version is biased. Nevertheless, the next result shows that the bias is of order $1 / N$, and is thus negligible compared to the standard deviation given in Theorem 3.1.

imsart-aos ver. 2010/04/27 file: cdfg.tex date: July 2, 2010 
Proposition 4. If $F$ is continuous, then we have

$$
N \frac{\mathbb{E}[\hat{p}]-p}{p} \underset{N \rightarrow+\infty}{\longrightarrow} n_{0} \frac{1-p_{0}}{p_{0}}
$$

Thus the bias is positive and of order $\frac{1}{N}$ when $N$ goes to infinity:

$$
\frac{\mathbb{E}[\hat{p}]-p}{p} \sim \frac{1}{N} \frac{n_{0}\left(1-p_{0}\right)}{p_{0}}
$$

Putting all things together, we can write the following expansion:

$$
\hat{p}=p\left(1+\frac{1}{\sqrt{N}} \sqrt{n_{0} \frac{1-p_{0}}{p_{0}}+\frac{1-r_{0}}{r_{0}}} Z+\frac{1}{N} n_{0} \frac{1-p_{0}}{p_{0}}+o_{\mathbb{P}}\left(\frac{1}{N}\right)\right),
$$

where $Z$ is a standard Gaussian variable.

\section{Remarks:}

1. If one does not want any bias, then the solution is to make a first run to compute the levels, and a second run to actually compute the probability. But it is clear from the above formula that, for the same computational cost, it is better in term of relative error to use directly our algorithm 2 and compute the levels on the fly.

2. In this regard the remark 3.2 page 489 of [3] might be misleading: if the levels are chosen from start, or using a preliminary run, then the resulting probability estimate is unbiased, even if the level crossing probabilities are indeed dependent (see Cérou et al. [6]).

3. It is worth mentioning that the bias is always positive, giving a slightly overvalued estimate. As rare event analysis usually deals with catastrophic events, it is not a bad thing that the real value be a bit lower than the provided estimate.

\section{Tuning the algorithm.}

4.1. Choice of the kernel $K$. There is no completely general method for finding the best transition kernel $K$ because it depends on the application. But in the very classical case of a Gibbs measure given by a bounded potential, we can use the Metropolis algorithm, as first proposed by Metropolis et al. [21], or the more general version later proposed by Hastings [15]. 
4.2. Less dependent sample. As mentioned in section 2.3, for the fixedlevels version of the algorithm we always have

$$
\sigma^{2} \geq \sum_{k=0}^{n-1} \frac{1-p_{k}}{p_{k}}
$$

The equality holds if and only if for all $k$, knowing that $X_{k-1} \in A_{k}$, one has

$$
\mathbb{P}\left(X_{n-1} \in A_{n} \mid X_{k}\right) \perp X_{k}
$$

To reach this goal, a simple idea is to iterate the transition kernel $M_{k}$ at each step as it provides more independence among particles.

The application of the kernel over the particles altogether can be seen as a kernel $\left(M_{k}\right)^{\otimes N}$, and the joint law of an i.i.d. sample $\left(\mu_{k}\right)^{\otimes N}$ is an invariant measure for $\left(M_{k}\right)^{\otimes N}$ according to Proposition 1. Then, from Meyn and Tweedie [22, Prop. 13.3.2], the total variation distance between the law of the sample and $\left(\mu_{k}\right)^{\otimes N}$ is non increasing as we iterate the kernel. So these iterations can only improve the independence of the sample, and thus decrease the variance.

We now focus on Metropolis-Hastings type kernels. We define these kernels as Tierney [26]: Denote $\lambda$ the Lebesgue measure $\mathbb{R}^{d}$ and assume that $\mu$ has a density with respect to $\lambda: \mu(d y)=f_{\mu}(y) \lambda(d y)$. We also need an instrumental kernel $Q$ with the same density assumption: $Q(x, d y)=q(x, y) \lambda(d y)$. Now define the Metropolis-Hastings ratio $\alpha$ by

$$
\alpha(x, y)= \begin{cases}\min \left(\frac{f_{\mu}(y) q(y, x)}{f_{\mu}(x) q(x, y)}, 1\right) & \text { if } f_{\mu}(x) q(x, y)>0 \\ 1 & \text { if } f_{\mu}(x) q(x, y)=0\end{cases}
$$

Let us now denote by $\ell$ the off-diagonal density

$$
\ell(x, y)= \begin{cases}q(x, y) \alpha(x, y) & \text { if } x \neq y \\ 0 & \text { if } x=y\end{cases}
$$

and $r(x)=1-\int_{\mathbb{R}^{d}} \ell(x, y) \lambda(d y)$. We say that $K$ is a Metropolis kernel if it is of the form

$$
K(x, d y)=\ell(x, y) \lambda(d y)+r(x) \delta_{x}(d y) .
$$

Note that in the framework of section 2.1, $M_{k}$ is a Metropolis-Hastings kernel as soon as $K$ is. 
We further assume that $K$ is $\mu$-irreducible, and that $\mu\left(\left\{x \in \mathbb{R}^{d}, r(x)>0\right\}\right)>$ 0 . This is usually the case, especially whenever $\forall(x, y) \in \mathbb{R}^{d} \times \mathbb{R}^{d}, q(x, y)>0$. Remark that if $K$ is $\mu$-irreducible, then $M_{k}$ is obviously $\mu_{k}$-irreducible ${ }^{1}$. Then, using Tierney [26, Corollary 2], they are also Harris recurrent, and by Theorem 13.3.3 in Meyn and Tweedie [22], any initial distribution $\nu$ such that $^{2} \nu\left(A_{k}\right)=1$ yields

$$
\left\|\int \nu(d x)\left(M_{k}\right)^{m}(x, .)-\mu_{k}\right\| \underset{m \rightarrow+\infty}{\longrightarrow} 0
$$

where the norm is in total variation. Then, for any initial cloud of particles $\Xi=\left(\xi^{1}, \ldots, \xi^{N}\right)$ and any test function $\left(\varphi_{1}, \ldots, \varphi_{N}\right)$,

$$
\begin{aligned}
& \left|\delta_{\Xi}\left(\left(M_{k}\right)^{\otimes N}\right)^{m}\left(\bigotimes_{j=1}^{N} \varphi_{j}\right)-\prod_{j=1}^{N} \mu_{k}\left(\varphi_{j}\right)\right| \\
& \quad=\left|\prod_{j=1}^{N}\left(M_{k}\right)^{m}\left(\varphi_{j}\right)\left(\xi^{j}\right)-\prod_{j=1}^{N} \mu_{k}\left(\varphi_{j}\right)\right| \underset{m \rightarrow+\infty}{\longrightarrow} 0 .
\end{aligned}
$$

For all test functions $\varphi$ on $\left(\mathbb{R}^{d}\right)^{N}$, a standard density argument gives

$$
\left|\delta_{\Xi}\left(\left(M_{k}\right)^{\otimes N}\right)^{m}(\varphi)-\mu_{k}^{\otimes N}(\varphi)\right| \underset{m \rightarrow+\infty}{\longrightarrow} 0 .
$$

This means that the more the kernel is iterated, the closer (in distribution) we get to an independent sample. Thus, at each step of Algorithm 2 (adaptive levels), we can think of iterating the kernel a fixed number of times (for example 20 times in the simulations of section 5.1).

4.3. Mixing property of the kernel $K$. We have written the algorithms using a unique kernel $K$ for all the iterations. Usually it is quite easy to construct not only one, but a family of kernels that are all $\mu$-symmetric, but with different mixing properties. This is useful when applying $K$ to the current particles, most of the transitions are refused (their scores are below the current threshold). In this case, we propose a change to another kernel which is less mixing, i.e. in some way with "smaller steps", and thus with a lower probability of going below the current level $L_{k}$. On the other hand, when almost all the transitions are accepted, it means that the kernel is poorly mixing, and that we could decrease the variance by choosing a kernel $K$ that is more mixing, i.e. with "larger steps". For example, this is tuned by the parameter $\alpha$ in section 5.1.

\footnotetext{
${ }^{1}$ As discussed in Tierney [26, page 1705], $K$ and $M_{k}$ are also aperiodic.

${ }^{2}$ This is only to avoid uninteresting pathological cases.
} 
5. Applications. Our motivation comes from problems occurring in the protection of digital contents. Here the term watermarking refers to a set of techniques for embedding/hiding information in a digital file (typically audio or video), such that the change is not perceptible, and very hard to remove. See the web site of the Copy Protection Working Group [8] for details.

In order to be used in an application, a watermarking technique must be reliable. Here are two application scenarii where a wrong estimation of the probability of error could lead to a disaster.

Copy protection.. Assume commercial contents are encrypted and watermarked and that future consumer electronics storage devices have a watermark detector. These devices refuse to record a watermarked content since it is copyrighted material. The probability of false alarm is the probability that the detector considers an original piece of content (which has not been watermarked) as protected. The movie that a user shot during his holidays could be rejected by his storage device. This absolutely non user-friendly behavior really scares consumer electronics manufacturers. In the past, the Copy Protection Working Group of the DVD forum evaluated that at most one false alarm should happen in 400 hours of video [8]. As the detection rate was one decision per ten seconds, this implies a probability of false alarm in the order of $10^{-5}$. An accurate experimental assessment of such a low probability of false alarm would demand to feed a real-time watermarking detector with non-watermarked content during 40,000 hours, i.e. more than 4 years! Proposals in response of the CPTWG's call were, at that time, never able to guarantee this level of reliability.

Fingerprinting.. In this application, users' identifiers are embedded in a purchased content. When this content is found in an illegal place (e.g. a P2P network), the copyright holders decode the hidden message, find an identifier, and thus they can trace the traitor, i.e. the customer who has illegally broadcast his copy. However, the task is not that simple because dishonest users might collude. For security reason, anti-collusion codes have to be employed. Yet, these solutions (also called weak traceability codes, see Barg et al. [2]) have a non-zero probability of error (defined as the probability of accusing an innocent). This probability should be, of course, extremely low, but it is also a very sensitive parameter: anti-collusion codes get longer (in terms of the number of bits to be hidden in content) as the probability of error decreases. Fingerprint designers have to strike a tradeoff, which is hard to conceive when only rough estimation of the probability of error is known. The major issue for fingerprinting algorithms is the fact that embedding large sequences implies also assessing reliability on a huge

imsart-aos ver. 2010/04/27 file: cdfg.tex date: July 2, 2010 
amount of data, which may be practically unachievable without using rare event analysis.

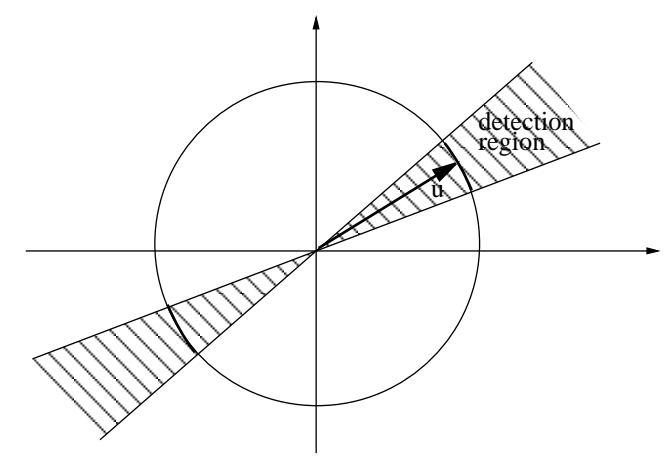

FIG 1. Detection region for zero-bit watermarking.

5.1. Zero-bit watermarking. In this example of zero-bit watermarking, $X$ is a Gaussian vector in $\mathbb{R}^{d}$, with zero mean and identity covariance matrix, $\Phi(X)=\frac{|\langle X, u\rangle|}{\|X\|}$ where $u$ is a fixed normalized vector (see Merhav and Sabbag [20]). Then the region $A=\left\{x \in \mathbb{R}^{d}\right.$ s.t. $\left.\Phi(x) \geq L\right\}$ is a cone as shown on figure 1. For a Gaussian distribution, the obvious choice for the kernel is the following: if we start from any point $x$, then the new position is given by

$$
x^{\prime}=\frac{x+\alpha W}{\sqrt{1+\alpha^{2}}}
$$

where $W$ is a $\mathcal{N}\left(0, I_{d}\right) \mathbb{R}^{d}$ valued random vector and $\alpha$ a positive number. This simple setup allows us to compare our estimates of the rare event probability with the result of a numerical integration. For example, in our simulations $d=20$ and $L=0.95$, so that $p=\mathbb{P}(\Phi(X) \geq L) \approx 4.70 \cdot 10^{-11}$. For such a low probability, it is of course out of question to run a classical Monte Carlo algorithm. Our algorithm with adaptive levels was run with 20 iterations of the kernel $M_{k}$ at each step. The choice of the mixing parameter $\alpha=0.3$ has experimentally proved to be a good trade-off, see discussion in section 4.3 above for details. The proportion of particles surviving from one step to another has been fixed to $p_{0}=0.75$, so that

$$
n_{0}=\left\lfloor\frac{\log p}{\log p_{0}}\right\rfloor=82 \text { and } r_{0}=p p_{0}^{-n_{0}} \approx 0.83
$$

For several numbers of particles, ranging from $N=100$ to $N=5,000$, we have run the algorithm 100 times in order to estimate the variance. Figure 2 


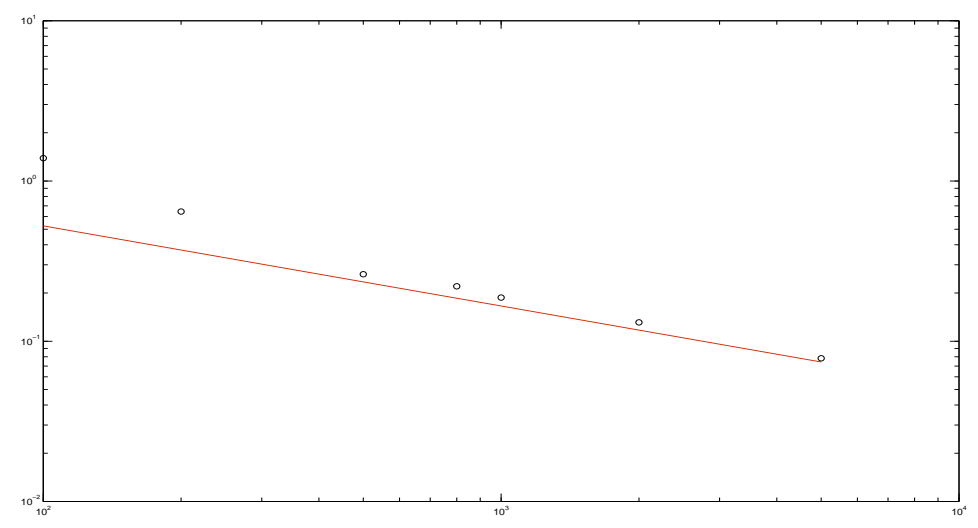

FIG 2. Theoretical and empirical relative standard deviations with 100 simulations.

shows in log-log plots the convergence of the normalized standard deviation to minimum achievable, which is that of i.i.d. samples at each stage, that is (see Theorem 3.1):

$$
\sqrt{\operatorname{Var}\left(\frac{\hat{p}-p}{p}\right)} \sim \frac{1}{\sqrt{N}} \sqrt{n_{0} \frac{1-p_{0}}{p_{0}}+\frac{1-r_{0}}{r_{0}}} .
$$

Clearly this indicates that even if we use the mixing kernel a finite number of times (here only 20 times), the empirical variance is on the same level as it would be for an independent sample (that is in the limit of infinite applications of the kernel), and therefore our theoretical results in this setting give a good picture of the accuracy of the actual algorithm.

Anyway, from a practical point of view, one would like to obtain an estimation of $p$ by running only one time the algorithm. In this aim, Theorem 3.1 and Proposition 4 allow us to construct confidence intervals. Indeed, we have

$$
\frac{\hat{p}-p}{p} \approx \mathcal{N}\left(\frac{n_{0}\left(1-p_{0}\right) / p_{0}}{N}, \frac{n_{0}\left(1-p_{0}\right) / p_{0}+\left(1-r_{0}\right) / r_{0}}{N}\right)
$$

so that an approximate $95 \%$ confidence interval for $p$ is given by $I=\left[\hat{p}_{-}, \hat{p}^{+}\right]$, where

$$
\hat{p}_{-}^{+}=\hat{p}\left(1-\frac{\hat{n}_{0}\left(1-p_{0}\right) / p_{0}}{N} \pm 2 \sqrt{\frac{\hat{n}_{0}\left(1-p_{0}\right) / p_{0}+\left(1-\hat{r}_{0}\right) / \hat{r}_{0}}{N}}\right) .
$$


This is illustrated on figure 3, where 100 such confidence intervals have been drawn for $N=500$ particles. In this example, 2 of them do not contain the true value $p=4.704 \cdot 10^{-11}$.

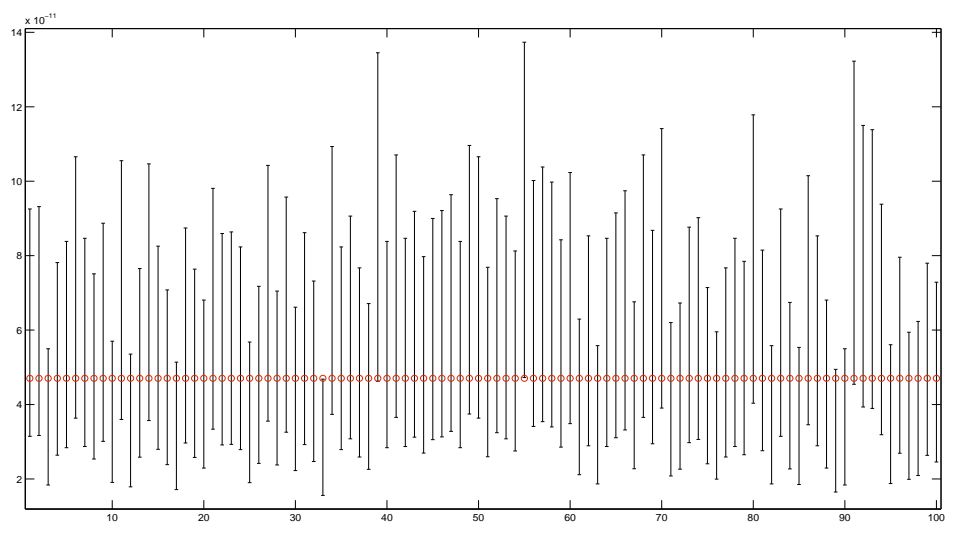

FIG 3. 95\% confidence intervals for $p=4.704 \cdot 10^{-11}$ with 100 simulations and $N=500$ particles.

5.2. Tardos probabilistic codes. We are interested here in embedding an identifier in each copy of a purchased content. Then a copy, which is the result of a collusion, is found on the web, and we want to decide whether or not it can be originated from a certain user. The rare event will be to consider an innocent as guilty.

The embedded message, called a fingerprint, consists of a sequence of bits $X=\left(X_{1}, \ldots, X_{m}\right)$, where each $X_{i}$ is independent from the others, and drawn from a Bernoulli's $\mathcal{B}\left(p_{i}\right)$. The $p_{i}$ 's are themselves i.i.d. random variables, drawn from a given distribution with density $f$ on $[0,1]$. Then we find a copy with fingerprint $y=\left(y_{1}, \ldots, y_{m}\right) \in\{0,1\}^{m}$. We conclude that a user is guilty if the score

$$
\Phi(X)=\sum_{i=1}^{m} y_{i} g_{i}\left(X_{i}\right)
$$

is larger than some value $L$, for some given functions $g_{i}$ 's. This approach was proposed by Tardos in [25], where he derives optimal choices for $f$ and the $g_{i}$ 's.

To apply our algorithm, we need to choose the kernel $K$. As the $X_{i}$ 's are independent, we randomly choose $r$ indices $\left\{j_{1}, \ldots, j_{r}\right\} \in\{1, \ldots, m\}$, with $r$ 


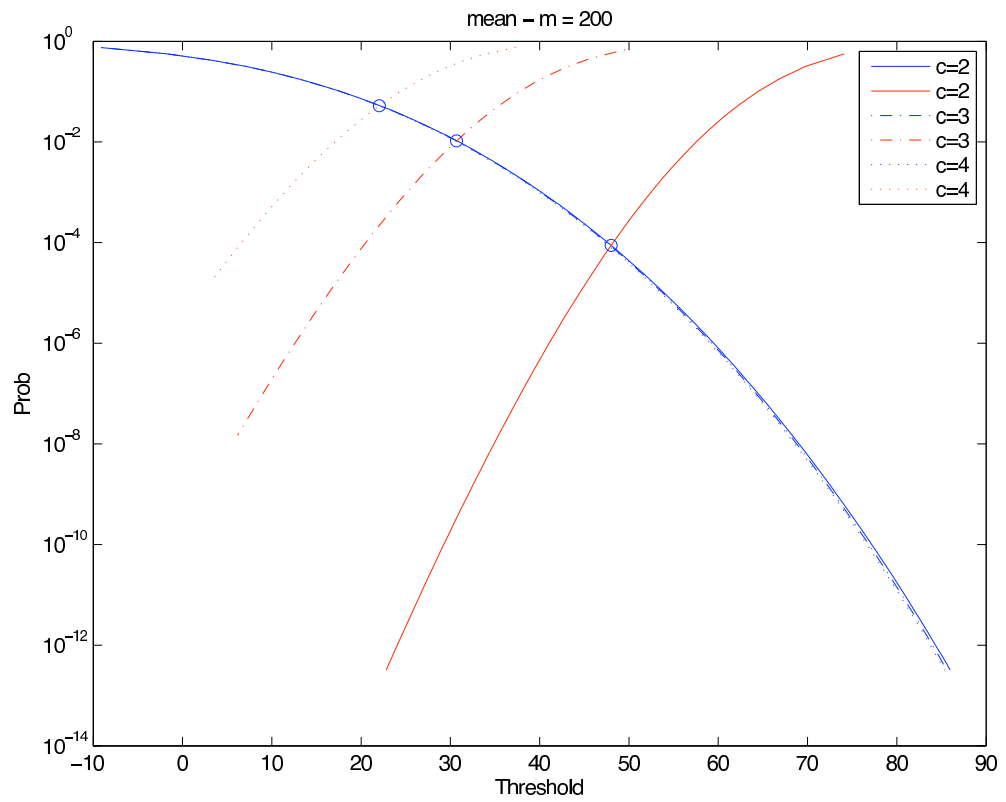

FIG 4. Mappings of the false positive probability (blue) and false negative probability (red) against the threshold. $m=200, c \in\{2,3,4\}$. The score of a particle is the mean of the $c$ colluders scores.

being a fixed parameter. Then for each $j_{\ell}$, we draw a new $X_{j_{\ell}}^{\prime}$ independently from the Bernoulli distribution $\mathcal{B}\left(p_{j_{\ell}}\right)$.

Using our adaptive algorithm, we made some numerical experiments on such codes. More precisely, we can easily estimate the probability of false detection (false positive) for some code length $m$, and collusion size $c$. The collusion strategy is to randomly pick up the symbols of pirated copy among the $c$ colluders' sequences. We can also estimate the probability of not accusing someone guilty (false negative). The results for $m=200$, and $c=2,3,4$ are shown on figure 4 . From these curves, one can then decide how to set the threshold $L$ to minimize the total error.

\section{APPENDIX A: PROOFS}

A.1. Proof of Proposition 1. We first prove that $\mu_{k}$ is invariant by the transition kernel $M_{k}$. We mainly use here the fact that the transition kernel 
$K$ is $\mu$-symmetric:

$$
\begin{aligned}
\int_{x} & \mu_{k}(d x) M_{k}(x, d y) \\
= & \int_{x} \mu_{k}(d x)\left(K(x, d y) \mathbb{1}_{A_{k}}(y)+K\left(x, A_{k}^{c}\right) \delta_{x}(d y)\right) \\
= & \int_{x} \int_{z} \mu_{k}(d x) K(x, d z)\left(\mathbb{1}_{A_{k}}(z) \delta_{z}(d y)+\mathbb{1}_{A_{k}^{c}}(z) \delta_{x}(d y)\right) \\
= & \int_{x} \frac{1}{\mu\left(A_{k}\right)} \mathbb{1}_{A_{k}}(x) \mu(d x) K(x, d y) \mathbb{1}_{A_{k}}(y) \\
& +\int_{z} \frac{1}{\mu\left(A_{k}\right)} \mathbb{1}_{A_{k}}(y) \mu(d y) K(y, d z) \mathbb{1}_{A_{k}^{c}}(z) \\
= & \int_{x} \frac{1}{\mu\left(A_{k}\right)} \mathbb{1}_{A_{k}}(x) \mu(d x) K(x, d y) \mathbb{1}_{A_{k}}(y) \\
& +\int_{z} \frac{1}{\mu\left(A_{k}\right)} \mathbb{1}_{A_{k}}(y) \mu(d z) K(z, d y) \mathbb{1}_{A_{k}^{c}}(z) \\
= & \mu_{k}(d y) .
\end{aligned}
$$

Now, in order to prove that $\mu$ is also invariant by the transition kernel $M_{k}$, we first remark that

$$
\mu(d x)=\mathbb{1}_{A_{k}^{c}}(x) \mu(d x)+\mathbb{1}_{A_{k}}(x) \mu(d x)=\mathbb{1}_{A_{k}^{c}}(x) \mu(d x)+\mu\left(A_{k}\right) \mu_{k}(d x) .
$$

Since we have

$$
\left.\int_{x} \mathbb{1}_{A_{k}^{c}}(x) \mu(d x) M_{k}(x, d y)=\int_{x} \mathbb{1}_{A_{k}^{c}}(x) \mu(d x) \delta_{x}(d y)\right)=\mathbb{1}_{A_{k}}(y) \mu(d y),
$$

and by the proof above

$$
\int_{x} \mu\left(A_{k}\right) \mu_{k}(d x) M_{k}(x, d y)=\mu\left(A_{k}\right) \mu_{k}(d y),
$$

we can conclude that

$$
\int_{x} \mu(d x) M_{k}(x, d y)=\mathbb{1}_{A_{k}}(y) \mu(d y)+\mu\left(A_{k}\right) \mu_{k}(d y)=\mu(d y),
$$

which ends the proof of Proposition 1. 
A.2. Proof of Proposition 2. We use induction to show that

$$
\mathbb{E}\left[\varphi\left(X_{k}\right) \prod_{m=0}^{k-1} \mathbb{1}_{A_{m+1}}\left(X_{m}\right)\right]=\mu\left(A_{k}\right) \mu_{k}(\varphi) .
$$

The case $k=0$ is obvious. Then assume the property be true for $k$. We write, using the Markov property and Proposition 1,

$$
\begin{aligned}
\mathbb{E} & {\left[\varphi\left(X_{k+1}\right) \prod_{m=0}^{k} \mathbb{1}_{A_{m+1}}\left(X_{m}\right)\right] } \\
= & \mathbb{E}\left[\mathbb{E}\left[\varphi\left(X_{k+1}\right) \mid X_{0}, \ldots, X_{k}\right] \mathbb{1}_{A_{k+1}}\left(X_{k}\right) \prod_{m=0}^{k-1} \mathbb{1}_{A_{m+1}}\left(X_{m}\right)\right] \\
= & \mu_{k}\left(M_{k+1}(\varphi) \mathbb{1}_{A_{k+1}}\right) \times \mu\left(A_{k}\right) \\
= & \left(\mathbb{1}_{A_{k}} \mu\right)\left(M_{k+1}(\varphi) \mathbb{1}_{A_{k+1}}\right) \\
= & \left(\mathbb{1}_{A_{k}} \mathbb{1}_{A_{k+1}} \mu\right)\left(M_{k+1}(\varphi)\right) \\
= & \mu_{k+1}\left(M_{k+1}(\varphi)\right) \times \mu\left(A_{k+1}\right) \\
= & \mu_{k+1}(\varphi) \times \mu\left(A_{k+1}\right) .
\end{aligned}
$$

Then taking the case $\varphi=\mathbb{1}$ we have

$$
\mathbb{E}\left[\prod_{m=0}^{k} \mathbb{1}_{A_{m+1}}\left(X_{m}\right)\right]=\mu\left(A_{k+1}\right),
$$

which concludes the proof.

A.3. Proof of Proposition 3. Adopting the notations of Proposition 9.4.1 page 301 of [9], the application of the first formula of page 304 leads to

$$
\operatorname{Var}(\hat{p})=\mathbb{E}\left[W_{n-1}^{\gamma}(1)^{2}\right]=\gamma_{n-1}^{2} \sum_{k=0}^{n-1} \eta_{k}\left(\left(\frac{Q_{k, n-1}(1)}{\eta_{k} Q_{k, n-1}(1)}-1\right)^{2}\right),
$$

In our context this can be rewritten as follows

$$
\begin{aligned}
\frac{\operatorname{Var}(\hat{p})}{p^{2}}= & \sum_{k=0}^{n-1} \mathbb{E}\left[\left(\frac{\mathbb{P}\left(X_{n-1} \in A_{n} \mid X_{k}\right)}{\mathbb{P}\left(X_{n-1} \in A_{n} \mid X_{k-1} \in A_{k}\right)}-1\right)^{2} \mid X_{k-1} \in A_{k}\right] \\
& \text { imsart-aos ver. 2010/04/27 file: cdfg.tex date: July 2, } 2010
\end{aligned}
$$


This leads to

$$
\frac{\operatorname{Var}(\hat{p})}{p^{2}}=\sum_{k=0}^{n-1}\left(\frac{\mathbb{E}\left[\mathbb{P}\left(X_{n-1} \in A_{n} \mid X_{k}\right)^{2} \mid X_{k-1} \in A_{k}\right]}{\mathbb{P}\left(X_{n-1} \in A_{n}\right)^{2}}-1\right) .
$$

Now one can readily check that

$$
\begin{aligned}
& \mathbb{E}\left[\mathbb{P}\left(X_{n-1} \in A_{n} \mid X_{k}\right)^{2} \mid X_{k-1} \in A_{k}\right]= \\
& \quad \mathbb{E}\left[\mathbb{P}\left(X_{n-1} \in A_{n} \mid X_{k}\right)^{2} \mathbb{1}_{A_{k+1}}\left(X_{k}\right) \mid X_{k-1} \in A_{k}\right],
\end{aligned}
$$

and this is equivalent to

$$
\begin{aligned}
& \mathbb{E}\left[\mathbb{P}\left(X_{n-1} \in A_{n} \mid X_{k}\right)^{2} \mid X_{k-1} \in A_{k}\right]= \\
& \frac{\mathbb{E}\left[\mathbb{P}\left(X_{n-1} \in A_{n} \mid X_{k}\right)^{2} \mathbb{1}_{A_{k+1}}\left(X_{k}\right) \mathbb{1}_{A_{k}}\left(X_{k-1}\right)\right]}{\mathbb{P}\left(X_{k-1} \in A_{k}\right)} .
\end{aligned}
$$

Since $\left\{X_{k} \in A_{k+1}\right\}$ implies $\left\{X_{k-1} \in A_{k}\right\}$, this last expression can be simplified

$$
\mathbb{E}\left[\mathbb{P}\left(X_{n-1} \in A_{n} \mid X_{k}\right)^{2} \mid X_{k-1} \in A_{k}\right]=\frac{\mathbb{E}\left[\mathbb{P}\left(X_{n-1} \in A_{n} \mid X_{k}\right)^{2} \mathbb{1}_{A_{k+1}}\left(X_{k}\right)\right]}{\mathbb{P}\left(X_{k-1} \in A_{k}\right)},
$$

and rewritten as

$$
\begin{aligned}
& \mathbb{E}\left[\mathbb{P}\left(X_{n-1} \in A_{n} \mid X_{k}\right)^{2} \mid X_{k-1} \in A_{k}\right]= \\
& \mathbb{E}\left[\mathbb{P}\left(X_{n-1} \in A_{n} \mid X_{k}\right)^{2} \mid X_{k} \in A_{k+1}\right] \times \frac{\mathbb{P}\left(X_{k} \in A_{k+1}\right)}{\mathbb{P}\left(X_{k-1} \in A_{k}\right)} .
\end{aligned}
$$

Since $p_{k}=\mathbb{P}\left(X_{k} \in A_{k+1} \mid X_{k-1} \in A_{k}\right)$ and $\left\{X_{k} \in A_{k+1}\right\} \Rightarrow\left\{X_{k-1} \in A_{k}\right\}$, we deduce

$$
\mathbb{P}\left(X_{n-1} \in A_{n} \mid X_{k-1} \in A_{k}\right)=p_{k} \mathbb{P}\left(X_{n-1} \in A_{n} \mid X_{k} \in A_{k+1}\right),
$$

so that

$\frac{\mathbb{E}\left[\mathbb{P}\left(X_{n-1} \in A_{n} \mid X_{k}\right)^{2} \mid X_{k-1} \in A_{k}\right]}{\mathbb{P}\left(X_{n-1} \in A_{n} \mid X_{k-1} \in A_{k}\right)^{2}}=\frac{\mathbb{E}\left[\mathbb{P}\left(X_{n-1} \in A_{n} \mid X_{k}\right)^{2} \mid X_{k} \in A_{k+1}\right]}{p_{k} \mathbb{P}\left(X_{n-1} \in A_{n} \mid X_{k} \in A_{k+1}\right)^{2}}$.

Now it remains to notice that

$$
\begin{aligned}
& \frac{\mathbb{E}\left[\mathbb{P}\left(X_{n-1} \in A_{n} \mid X_{k}\right)^{2} \mid X_{k} \in A_{k+1}\right]}{\mathbb{P}\left(X_{n-1} \in A_{n} \mid X_{k} \in A_{k+1}\right)^{2}}= \\
& 1+\mathbb{E}\left[\left(\frac{\mathbb{P}\left(X_{n-1} \in A_{n} \mid X_{k}\right)}{\mathbb{P}\left(X_{n-1} \in A_{n} \mid X_{k} \in A_{k+1}\right)}-1\right)^{2} \mid X_{k} \in A_{k+1}\right], \\
& \quad \text { imsart-aos ver. 2010/04/27 file: cdfg.tex date: July 2, } 2010
\end{aligned}
$$


so that coming back to (A.1) gives the desired result

$$
\begin{aligned}
\frac{\operatorname{Var}(\hat{p})}{p^{2}}= & \sum_{k=0}^{n-1} \frac{1-p_{k}}{p_{k}}+ \\
& \sum_{k=0}^{n-1} \frac{1}{p_{k}} \mathbb{E}\left[\left(\frac{\mathbb{P}\left(X_{n-1} \in A_{n} \mid X_{k}\right)}{\mathbb{P}\left(X_{n-1} \in A_{n} \mid X_{k-1} \in A_{k}\right)}-1\right)^{2} \mid X_{k-1} \in A_{k}\right] .
\end{aligned}
$$

A.4. Proof of Theorem 3.1. In order to simplify the writings, we will suppose that $p_{0} N$ is an integer. Then, for all real numbers $L$ and $L^{\prime}$ such that $L<L^{\prime}$, let us denote

$$
F\left(L, L^{\prime}\right)=\mathbb{P}\left(\Phi(X) \leq L^{\prime} \mid \Phi(X)>L\right)=\frac{F\left(L^{\prime}\right)-F(L)}{1-F(L)},
$$

with the convention that $F\left(L, L^{\prime}\right)=0$ if $F(L)=1$.

We first notice the following crucial point: given $\hat{L}_{k}$, the random vectors $\xi_{k}^{j}$, for $j \in\{1, \ldots, N\}$, are i.i.d., and thus so are the random variables $\Phi\left(\xi_{k}^{j}\right)$. Since $F$ is continuous, given $\hat{L}_{k}$, the random variable $F\left(\hat{L}_{k}, \hat{L}_{k+1}\right)$ has the same distribution as the random variable $U_{\left(\left(1-p_{0}\right) N\right)}$, where $\left(U_{i}\right)_{1 \leq i \leq N}$ is a triangular array of identically distributed random variables with uniform law on $[0,1]$ and row-wise independent, and for all $N \geq 1$

$$
U_{(1)} \leq U_{(2)} \leq \ldots \leq U_{(N)} .
$$

Let us denote $G_{N}$ the empirical cdf of $\left(U_{i}\right)_{1 \leq i \leq N}$ and $G(x)=x$ the cdf of the uniform law on $[0,1]$. Then from the basic identities $\left\|G_{N}-G\right\|_{\infty}=$ $\left\|G_{N}^{-1}-G\right\|_{\infty}$ and $U_{\left(\left(1-p_{0}\right) N\right)}=G_{N}^{-1}\left(1-p_{0}\right)$, we can deduce that

$$
\left|U_{\left(\left(1-p_{0}\right) N\right)}-\left(1-p_{0}\right)\right| \leq\left\|G_{N}-G\right\|_{\infty} .
$$

Using Dvoretzky-Kiefer-Wolfowitz (DKW) inequality (see for example [27]), we have then for all $\varepsilon>0$

$$
\mathbb{P}\left(\left\|G_{N}-G\right\|_{\infty}>\varepsilon\right) \leq 2 \exp \left(-2 N \varepsilon^{2}\right),
$$

hence

$$
\begin{aligned}
\mathbb{P}\left(\left|F\left(\hat{L}_{k}, \hat{L}_{k+1}\right)-\left(1-p_{0}\right)\right|>\varepsilon\right) & =\mathbb{P}\left(\left|U_{\left(\left(1-p_{0}\right) N\right)}-\left(1-p_{0}\right)\right|>\varepsilon\right) \\
& \leq 2 \exp \left(-2 N \varepsilon^{2}\right)
\end{aligned}
$$

Using Borel-Cantelli lemma, we conclude that

$$
\begin{aligned}
& F\left(\hat{L}_{k}, \hat{L}_{k+1}\right) \underset{N \rightarrow \infty}{\stackrel{\text { a.s. }}{\longrightarrow}} 1-p_{0} . \\
& \text { imsart-aos ver. } 2010 / 04 / 27 \text { file: cdfg.tex date: July 2, } 2010
\end{aligned}
$$


From the theory of order statistics (see for example [1], Theorem 8.5.1, p. 223), we also deduce the convergence in distribution

$$
\sqrt{N}\left(1-F\left(\hat{L}_{k}, \hat{L}_{k+1}\right)-p_{0}\right) \underset{N \rightarrow \infty}{\stackrel{\mathcal{D}}{\longrightarrow}} \mathcal{N}\left(0, p_{0}\left(1-p_{0}\right)\right) .
$$

To prove the result of Theorem 3.1, we proceed by induction, assuming that:

$$
\sqrt{N}\left(\prod_{m=1}^{k}\left(1-F\left(\hat{L}_{m-1}, \hat{L}_{m}\right)\right)-p_{0}^{k}\right) \underset{N \rightarrow \infty}{\stackrel{\mathcal{D}}{\longrightarrow}} \mathcal{N}\left(0, \sigma_{k}^{2}\right) .
$$

For the next step, we use the decomposition

$$
\begin{aligned}
& \sqrt{N}\left(\prod_{m=1}^{k+1}\left(1-F\left(\hat{L}_{m-1}, \hat{L}_{m}\right)\right)-p_{0}^{k+1}\right) \\
& \text { ₹A. } 4) \bar{N}\left(\prod_{m=1}^{k}\left(1-F\left(\hat{L}_{m-1}, \hat{L}_{m}\right)\right)-p_{0}^{k}\right)\left(1-F\left(\hat{L}_{k}, \hat{L}_{k+1}\right)-p_{0}\right) \\
& \quad+p_{0} \sqrt{N}\left(\prod_{m=1}^{k}\left(1-F\left(\hat{L}_{m-1}, \hat{L}_{m}\right)\right)-p_{0}^{k}\right)+p_{0}^{k} \sqrt{N}\left(1-F\left(\hat{L}_{k}, \hat{L}_{k+1}\right)-p_{0}\right) .
\end{aligned}
$$

The almost sure convergence of equation (A.2) and the induction hypothesis ensure that the first term converges in probability to 0 when $N$ goes to infinity. To prove the convergence in distribution of the other terms of (A.4), let us introduce the characteristic function

$$
\begin{aligned}
\phi_{N}(t)= & \mathbb{E}\left[\operatorname { e x p } \left(i t \left(p_{0} \sqrt{N}\left(\prod_{m=1}^{k}\left(1-F\left(\hat{L}_{m-1}, \hat{L}_{m}\right)\right)-p_{0}^{k}\right)\right.\right.\right. \\
& \left.\left.+p_{0}^{k} \sqrt{N}\left(1-F\left(\hat{L}_{k}, \hat{L}_{k+1}\right)-p_{0}\right)\right)\right]
\end{aligned}
$$

Conditioning with respect to $\hat{L}_{1}, \ldots, \hat{L}_{k}$ gives

$$
\begin{aligned}
& \phi_{N}(t)= \mathbb{E}\left[\exp \left(i t\left(p_{0} \sqrt{N}\left(\prod_{m=1}^{k}\left(1-F\left(\hat{L}_{m-1}, \hat{L}_{m}\right)\right)-p_{0}^{k}\right)\right)\right)\right. \\
& \times \mathbb{E}\left[\exp \left(i t\left(p_{0}^{k} \sqrt{N}\left(1-F\left(\hat{L}_{k}, \hat{L}_{k+1}\right)-p_{0}\right)\right) \mid \hat{L}_{1}, \ldots, \hat{L}_{k}\right]\right] .
\end{aligned}
$$

Thanks to the strong Markov property of the $\hat{L}_{k}$ 's, this can be reduced to

$$
\begin{aligned}
\phi_{N}(t)= & \mathbb{E}\left[\exp \left(i t\left(+p_{0} \sqrt{N}\left(\prod_{m=1}^{k}\left(1-F\left(\hat{L}_{m-1}, \hat{L}_{m}\right)\right)-p_{0}^{k}\right)\right)\right)\right. \\
& \times \mathbb{E}\left[\exp \left(i t\left(p_{0}^{k} \sqrt{N}\left(1-F\left(\hat{L}_{k}, \hat{L}_{k+1}\right)-p_{0}\right)\right) \mid \hat{L}_{k}\right]\right], \\
& \text { imsart-aos ver. 2010/04/27 file: cdfg.tex date: July 2, } 2010
\end{aligned}
$$


and we can remark that

$$
\begin{array}{r}
\mathbb{E}\left[\exp \left(i t\left(p_{0}^{k} \sqrt{N}\left(1-F\left(\hat{L}_{k}, \hat{L}_{k+1}\right)-p_{0}\right)\right) \mid \hat{L}_{k}\right]\right. \\
\quad=\mathbb{E}\left[\exp \left(i t\left(p_{0}^{k} \sqrt{N}\left(1-U_{\left(\left(1-p_{0}\right) N\right)}-p_{0}\right)\right)\right],\right.
\end{array}
$$

where $U_{\left(\left(1-p_{0}\right) N\right)}$ is independent of $\hat{L}_{1}, \ldots, \hat{L}_{k}$. This leads to

$$
\begin{aligned}
\phi_{N}(t)= & \mathbb{E}\left[\exp \left(i t\left(+p_{0} \sqrt{N}\left(\prod_{m=1}^{k}\left(1-F\left(\hat{L}_{m-1}, \hat{L}_{m}\right)\right)-p_{0}^{k}\right)\right)\right)\right] \\
& \times \mathbb{E}\left[\exp \left(i t\left(p_{0}^{k} \sqrt{N}\left(1-U_{\left(\left(1-p_{0}\right) N\right)}-p_{0}\right)\right)\right] .\right.
\end{aligned}
$$

Thanks to the induction hypothesis and to equation (A.3), it comes

$$
\begin{aligned}
& p_{0} \sqrt{N}\left(\prod_{m=1}^{k}\left(1-F\left(\hat{L}_{m-1}, \hat{L}_{m}\right)\right)-p_{0}^{k}\right) \\
&+p_{0}^{k} \sqrt{N}\left(1-F\left(\hat{L}_{k}, \hat{L}_{k+1}\right)-p_{0}\right) \\
& \quad \underset{N \rightarrow \infty}{\stackrel{\mathcal{D}}{\longrightarrow}} \mathcal{N}\left(0, p_{0}^{2} \sigma_{k}^{2}+p_{0}^{2 k+1}\left(1-p_{0}\right)\right) .
\end{aligned}
$$

Putting all pieces together gives finally

$$
\sqrt{N}\left(\prod_{m=1}^{k+1}\left(1-F\left(\hat{L}_{m-1}, \hat{L}_{m}\right)\right)-p_{0}^{k+1}\right) \underset{N \rightarrow \infty}{\stackrel{\mathcal{D}}{\longrightarrow}} \mathcal{N}\left(0, \sigma_{k+1}^{2}\right)
$$

with $\sigma_{k+1}^{2}=p_{0}^{2} \sigma_{k}^{2}+p_{0}^{2 k+1}\left(1-p_{0}\right)$. From this recursion we deduce that for all $k \geq 1$

$$
\sigma_{k}^{2}=k p_{0}^{2 k-1}\left(1-p_{0}\right) .
$$

It remains to deal with the last step. For the sake of simplicity, we suppose that $\log p / \log p_{0}$ is not an integer. Let us first consider an alternative algorithm defined as follows: we run algorithm 2 with the deterministic number of steps $n_{0}$ and denote $\hat{p}_{d}=\hat{r}_{d} p_{0}^{n_{0}}$ the corresponding estimator, where

$$
\hat{r}_{d}=\frac{1}{N} \sum_{j=1}^{N} \mathbb{1}_{\left\{\Phi\left(\xi_{n_{0}}^{j}\right) \geq L\right\}} .
$$

In this expression, knowing $\hat{L}_{n_{0}}$, the random variables $\left(\mathbb{1}_{\left\{\Phi\left(\xi_{n_{0}}^{j}\right) \geq L\right\}}\right)_{1 \leq j \leq N}$ are i.i.d. Bernoulli trials with parameter

$$
\begin{array}{r}
r=\mathbb{P}\left(\mathbb{1}_{\left\{\Phi\left(\xi_{n_{0}}^{j}\right) \geq L\right\}}=1 \mid \hat{L}_{n_{0}}\right)=1-F\left(\hat{L}_{n_{0}}, L\right)=\frac{p}{1-F\left(\hat{L}_{n_{0}}\right)}=\frac{r_{0} p_{0}^{n_{0}}}{1-F\left(\hat{L}_{n_{0}}\right)} . \\
\text { imsart-aos ver. } 2010 / 04 / 27 \text { file: cdfg.tex date: July 2, } 2010
\end{array}
$$


Then we can write

$$
\sqrt{N}\left(r_{0}-r\right)=\sqrt{N}\left(1-F\left(\hat{L}_{n_{0}}\right)-p_{0}^{n_{0}}\right) \frac{r_{0}}{1-F\left(\hat{L}_{n_{0}}\right)} .
$$

Now we use the almost sure convergence

$$
1-F\left(\hat{L}_{n_{0}}\right) \underset{N \rightarrow \infty}{\stackrel{a . s .}{\longrightarrow}} p_{0}^{n_{0}},
$$

and the convergence in distribution from equation (A.5)

$$
\sqrt{N}\left(1-F\left(\hat{L}_{n_{0}}\right)-p_{0}^{n_{0}}\right) \underset{N \rightarrow \infty}{\stackrel{\mathcal{D}}{\longrightarrow}} \mathcal{N}\left(0, \sigma_{n_{0}}^{2}\right),
$$

to conclude that

$$
\sqrt{N}\left(r_{0}-r\right) \underset{N \rightarrow \infty}{\stackrel{\mathcal{D}}{\longrightarrow}} \mathcal{N}\left(0, n_{0} \frac{1-p_{0}}{p_{0}} r_{0}^{2}\right) .
$$

From this we deduce that

$$
\sqrt{N}\left(r_{0}-\hat{r}_{d}\right) \underset{N \rightarrow \infty}{\stackrel{\mathcal{D}}{\longrightarrow}} \mathcal{N}\left(0, n_{0} \frac{1-p_{0}}{p_{0}} r_{0}^{2}+\frac{1-r_{0}}{r_{0}}\right) .
$$

Since $\hat{p}_{d}=\hat{r}_{d} p_{0}^{n_{0}}$, it comes

$$
\sqrt{N}\left(\hat{p}_{d}-p\right) \underset{N \rightarrow \infty}{\stackrel{\mathcal{D}}{\longrightarrow}} \mathcal{N}\left(0, p^{2}\left(n_{0} \frac{1-p_{0}}{p_{0}}+\frac{1-r_{0}}{r_{0}}\right)\right) .
$$

Coming back to the "true" estimator $\hat{p}$, we have

$$
\sqrt{N}(\hat{p}-p)=\sqrt{N}(\hat{p}-p) \mathbb{1}_{\hat{n}_{0}=n_{0}}+\sqrt{N}(\hat{p}-p) \mathbb{1}_{\hat{n}_{0} \neq n_{0}},
$$

but one can readily see that $\hat{p} \mathbb{1}_{\hat{n}_{0}=n_{0}}=\hat{p}_{d} \mathbb{1}_{\hat{n}_{0}=n_{0}}$ almost surely, so that

$$
\sqrt{N}(\hat{p}-p)=\sqrt{N}\left(\hat{p}_{d}-p\right)+\sqrt{N}\left(\hat{p}-\hat{p}_{d}\right) \mathbb{1}_{\hat{n}_{0} \neq n_{0}},
$$

and the proof of Theorem 3.1 will be complete if we show that

$$
\sqrt{N}\left(\hat{p}-\hat{p}_{d}\right) \mathbb{1}_{\hat{n}_{0} \neq n_{0}} \underset{N \rightarrow \infty}{\stackrel{\mathbb{P}}{\longrightarrow}} 0 .
$$

In this aim, let us first remark that for all $\varepsilon>0$

$$
\mathbb{P}\left(\left|\sqrt{N}(p-\hat{p}) \mathbb{1}_{\hat{n}_{0} \neq n_{0}}\right|>\varepsilon\right) \leq \mathbb{P}\left(\hat{n}_{0} \neq n_{0}\right),
$$

then the following lemma allows us to conclude. 
Lemma 1. Denoting $c=\min \left(p_{0}-p^{\frac{1}{n_{0}}}, p^{\frac{1}{n_{0}+1}}-p_{0}\right)$, we have

$$
\mathbb{P}\left(\hat{n}_{0} \neq n_{0}\right) \leq 2\left(n_{0}+1\right) e^{-2 N c^{2}} .
$$

As a consequence

$$
\hat{n}_{0} \underset{N \rightarrow \infty}{\stackrel{a . s .}{\longrightarrow}} n_{0} .
$$

Proof. Let us denote $B=\left\{\hat{n}_{0}=n_{0}\right\}$ the event for which the algorithm stops after the correct number of steps. The following equalities are straightforward:

$$
\begin{aligned}
B & =\left\{\hat{L}_{n_{0}} \leq L<\hat{L}_{n_{0}+1}\right\} \\
& =\left\{1-F\left(\hat{L}_{n_{0}+1}\right)<1-F(L) \leq 1-F\left(\hat{L}_{n_{0}}\right)\right\} \\
& =\left\{\prod_{m=1}^{n_{0}+1}\left(1-F\left(\hat{L}_{m-1}, \hat{L}_{m}\right)\right)<p \leq \prod_{m=1}^{n_{0}}\left(1-F\left(\hat{L}_{m-1}, \hat{L}_{m}\right)\right)\right\} .
\end{aligned}
$$

For all $m=1, \ldots, n_{0}+1$, if we denote

$$
B_{m}=\left\{p^{\frac{1}{n_{0}}}-p_{0}<1-p_{0}-F\left(\hat{L}_{m-1}, \hat{L}_{m}\right)<p^{\frac{1}{n_{0}+1}}-p_{0}\right\}
$$

we have

$$
\begin{aligned}
\mathbb{P}(B) & \geq \mathbb{P}\left(B_{1} \cap \cdots \cap B_{n_{0}+1}\right) \\
& \geq 1-\sum_{m=1}^{n_{0}+1}\left(1-\mathbb{P}\left(B_{m}\right)\right) .
\end{aligned}
$$

Denoting $c=\min \left(p_{0}-p^{\frac{1}{n_{0}}}, p^{\frac{1}{n_{0}+1}}-p_{0}\right)$, the DKW inequality implies

$$
1-\mathbb{P}\left(B_{m}\right) \leq \mathbb{P}\left(\left|1-p_{0}-F\left(\hat{L}_{m-1}, \hat{L}_{m}\right)\right|>c\right) \leq 2 e^{-2 N c^{2}},
$$

so that the result of Lemma 1 is proved

$$
\mathbb{P}(B)=\mathbb{P}\left(\hat{n}_{0}=n_{0}\right) \geq 1-2\left(n_{0}+1\right) e^{-2 N c^{2}} .
$$


A.5. Proof of Proposition 4. As for the analysis of the standard deviation, we first notice that

$$
N(\hat{p}-p)=N\left(\hat{p}_{d}-p\right)+N\left(\hat{p}-\hat{p}_{d}\right) \mathbb{1}_{\hat{n}_{0} \neq n_{0}},
$$

and applying Lemma 1 yields

$$
N \mathbb{E}\left[\left|\hat{p}-\hat{p}_{d}\right| \mathbb{1}_{\hat{n}_{0} \neq n_{0}}\right] \leq N \mathbb{P}\left(\hat{n}_{0} \neq n_{0}\right) \underset{N \rightarrow \infty}{\longrightarrow} 0,
$$

so that only the first term of the right-hand-side of equation (A.7) is worth considering for the convergence of $N \mathbb{E}[\hat{p}-p]$. Recall that the estimate is then $\hat{p}_{d}=\hat{r}_{d} p_{0}^{n_{0}}$, where $\hat{r}_{d}$ is defined as in equation (A.6), so that

$$
\mathbb{E}\left[\hat{r}_{d}\right]=\mathbb{E}\left[\mathbb{E}\left[\hat{r}_{d} \mid \hat{L}_{n_{0}}\right]\right]=\mathbb{E}[r]=\mathbb{E}\left[\frac{p}{1-F\left(\hat{L}_{n_{0}}\right)}\right] .
$$

Then the normalized bias is

$$
\begin{aligned}
\frac{\mathbb{E}[\hat{p}]-p}{p} & =\mathbb{E}\left[\frac{p_{0}^{n_{0}}}{1-F\left(\hat{L}_{n_{0}}\right)}\right]-1 \\
& =\mathbb{E}\left[\frac{F\left(\hat{L}_{n_{0}}\right)-F\left(L_{n_{0}}\right)}{1-F\left(\hat{L}_{n_{0}}\right)}\right] \\
& =\mathbb{E}\left[\frac{W}{a-W}\right]
\end{aligned}
$$

with $W=F\left(\hat{L}_{n_{0}}\right)-F\left(L_{n_{0}}\right)=F\left(\hat{L}_{n_{0}}\right)-\left(1-p_{0}^{n_{0}}\right)$, and $a=1-F\left(L_{n_{0}}\right)=p_{0}^{n_{0}}$. If we remark that

$$
1-F\left(\hat{L}_{n_{0}}\right)=\prod_{k=0}^{n_{0}-1}\left(1-F\left(\hat{L}_{k}, \hat{L}_{k+1}\right)\right)
$$

with the convention $\hat{L}_{0}=-\infty$, then the result of equation (A.2) implies

$$
\frac{W}{a}=\frac{F\left(\hat{L}_{n_{0}}\right)-\left(1-p_{0}^{n_{0}}\right)}{p_{0}^{n_{0}}} \underset{N \rightarrow+\infty}{\stackrel{a . s .}{\longrightarrow}} 0 .
$$

We may now rewrite

$$
\frac{\mathbb{E}[\hat{p}]-p}{p}=\frac{1}{a} \mathbb{E}\left[W \frac{1}{1-\frac{W}{a}}\right],
$$

and the asymptotic expansion $(1-x)^{-1}=1+x+o(x)$ leads to

$$
\frac{\mathbb{E}[\hat{p}]-p}{p}=\frac{1}{a} \mathbb{E}[W]+\frac{1}{a^{2}} \mathbb{E}\left[W^{2}\right]+\frac{1}{a^{2}} o\left(\mathbb{E}\left[W^{2}\right]\right) .
$$

Then we will use the following lemma. 
LEMMA 2.

$$
\mathbb{E}[W]=\mathbb{E}\left[F\left(\hat{L}_{n_{0}}\right)-\left(1-p_{0}^{n_{0}}\right)\right]=0
$$

and

$$
\mathbb{E}\left[W^{2}\right]=\operatorname{Var}\left(F\left(\hat{L}_{n_{0}}\right)-F\left(L_{n_{0}}\right)\right)=\frac{n_{0}}{N} p_{0}^{2 n_{0}-1}\left(1-p_{0}\right)+o\left(\frac{1}{N}\right) .
$$

The proof of this lemma is left to the next subsection. Coming back to (A.8), we have obtained

$$
\frac{\mathbb{E}[\hat{p}]-p}{p}=\frac{1}{N}\left(n_{0} \frac{1-p_{0}}{p_{0}}\right)+o\left(\frac{1}{N}\right),
$$

which ends the proof of Proposition 4.

A.6. Proof of Lemma 2. First of all, some notation. Let $\left(U_{i}\right)_{1 \leq i \leq N}$ be an i.i.d. family of random variables uniformly distributed on $(0,1)$. We denote by $U_{(i)}$ the $i$ th largest sample: $0 \leq U_{(1)} \leq \cdots \leq U_{(N)} \leq 1$. For simplicity, we will assume that $p_{0}=\frac{N_{0}}{N}$ for some $1 \leq N_{0} \leq N$. Then it is well known from the theory of order statistics (see for example e.g. [1, formula (2.2.20) page 14]) that

$$
\mathbb{E}\left[U_{\left(N-N_{0}\right)}\right]=1-p_{0}
$$

Expectation of $W$.. We will prove that it is equal to zero by induction on $n_{0}$. For $n_{0}=1, F\left(\hat{L}_{1}\right)$ has the same law as $U_{\left(N-N_{0}\right)}$, thus the result is obvious by equation (A.9). Now assume that $\mathbb{E}\left[F\left(\hat{L}_{n_{0}-1}\right)\right]=1-p_{0}^{n_{0}-1}$. From the decomposition

$$
\prod_{k=1}^{n_{0}}\left(1-F\left(\hat{L}_{k-1}, \hat{L}_{k}\right)\right)=1-F\left(\hat{L}_{n_{0}}\right)
$$

we deduce

$$
\begin{aligned}
\mathbb{E}\left[1-F\left(\hat{L}_{n_{0}}\right)\right] & =\mathbb{E}\left[\prod_{k=1}^{n_{0}}\left(1-F\left(\hat{L}_{k-1}, \hat{L}_{k}\right)\right)\right] \\
& =\mathbb{E}\left[\mathbb{E}\left[1-F\left(\hat{L}_{n_{0}-1}, \hat{L}_{n_{0}}\right) \mid \hat{L}_{n_{0}-1}\right] \prod_{k=1}^{n_{0}-1}\left(1-F\left(\hat{L}_{k-1}, \hat{L}_{k}\right)\right)\right] .
\end{aligned}
$$

Since

$$
\mathbb{E}\left[1-F\left(\hat{L}_{n_{0}-1}, \hat{L}_{n_{0}}\right) \mid \hat{L}_{n_{0}-1}\right]=\mathbb{E}\left[1-U_{\left(N-N_{0}\right)}\right]=p_{0},
$$

the induction property for $n_{0}-1$ implies

$$
\mathbb{E}\left[1-F\left(\hat{L}_{n_{0}}\right)\right]=p_{0} \mathbb{E}\left[\prod_{k=1}^{n_{0}-1}\left(1-F\left(\hat{L}_{k-1}, \hat{L}_{k}\right)\right)\right]=p_{0}^{n_{0}}
$$

which proves that $W$ has zero mean. 
Variance of $W . . \quad$ From the proof of Theorem 3.1, we know that

$$
\sqrt{N}\left(\prod_{k=1}^{n_{0}}\left(1-F\left(\hat{L}_{k-1}, \hat{L}_{k}\right)\right)-p_{0}^{n_{0}}\right) \underset{N \rightarrow+\infty}{\stackrel{\mathcal{D}}{\longrightarrow}} \mathcal{N}\left(0, \sigma_{n_{0}}^{2}\right),
$$

where $\sigma_{n_{0}}^{2}=n_{0}\left(1-p_{0}\right) p_{0}^{2 n_{0}-1}$. So we have

$$
\sqrt{N}\left(1-F\left(\hat{L}_{n_{0}}\right)-p_{0}^{n_{0}}\right) \underset{N \rightarrow+\infty}{\stackrel{\mathcal{D}}{\longrightarrow}} \mathcal{N}\left(0, \sigma_{n_{0}}^{2}\right),
$$

and by symmetry,

$$
\sqrt{N}\left(F\left(\hat{L}_{n_{0}}\right)-F\left(L_{n_{0}}\right)\right) \underset{N \rightarrow+\infty}{\stackrel{\mathcal{D}}{\longrightarrow}} \mathcal{N}\left(0, \sigma_{n_{0}}^{2}\right) .
$$

It means that

$$
\operatorname{Var}\left(F\left(\hat{L}_{n_{0}}\right)-F\left(L_{n_{0}}\right)\right)=\frac{1}{N} \sigma_{n_{0}}^{2}+o\left(\frac{1}{N}\right),
$$

which concludes the proof of Lemma 2.

\section{REFERENCES}

[1] Arnold, B. C., Balakrishnan, N. and Nagaraja, H. N. (1992). A first course in order statistics. Wiley Series in Probability and Mathematical Statistics: Probability and Mathematical Statistics. John Wiley \& Sons Inc., New York. A Wiley-Interscience Publication. MR1178934 (94a:62076)

[2] Barg, A., Blakley, G. R. and Kabatiansky, G. A. (2003). Digital fingerprinting codes: problem statements, constructions, identification of traitors. IEEE Trans. on Signal Processing $\mathbf{5 1}$ 960-980.

[3] Botev, Z. I. and Kroese, D. P. (2008). An Efficient Algorithm for Rare-event Probability Estimation, Combinatorial Optimization, and Counting. Methodology and Computing in Applied Probability 10 471-505.

[4] Bucklew, J. A. (2004). Introduction to rare event simulation. Springer Series in Statistics. Springer-Verlag, New York. MR2045385 (2005e:62001)

[5] Cérou, F., Del Moral, P. and Guyader, A. (2010). A non asymptotic variance theorem for unnormalized Feynman-Kac particle models. Annales de l'IHP (to appear).

[6] Cérou, F., Del Moral, P., Le Gland, F. and Lezaud, P. (2006). Genetic Genealogical Models in Rare Event Analysis. Latin American Journal of Probability and Mathematical Statistics 1.

[7] Cérou, F. and Guyader, A. (2007). Adaptive multilevel splitting for rare event analysis. Stoch. Anal. Appl. 25 417-443. MR2303095

[8] Copy Protection Technical Working Group, www.cptwg.org.

[9] Del Moral, P. (2004). Feynman-Kac formulae, Genealogical and interacting particle systems with applications. Probability and its Applications. Springer-Verlag, New York. MR2044973 (2005f:60003)

[10] Del Moral, P., Doucet, A. and Jasra, A. (2006). Sequential Monte Carlo samplers. J. R. Stat. Soc. Ser. B Stat. Methodol. 68 411-436. MR2278333 
[11] Del Moral, P. and Lezaud, P. (2006). Branching and interacting particle interpretation of rare event probabilities. In Stochastic Hybrid Systems : Theory and Safety Critical Applications, (H. Blom and J. Lygeros, eds.). Lecture Notes in Control and Information Sciences 337 277-323. Springer-Verlag, Berlin.

[12] Doucet, A., de Freitas, N. and Gordon, N., eds. (2001). Sequential Monte Carlo Methods in Practice. Statistics for Engineering and Information Science. SpringerVerlag, New York.

[13] Garvels, M. J. J. (2000). The splitting method in rare event simulation Thesis, University of Twente, Twente.

[14] Glasserman, P., Heidelberger, P., Shahabuddin, P. and Zajic, T. (1999). Multilevel splitting for estimating rare event probabilities. Oper. Res. 47 585-600. MR1710951 (2000e:60162)

[15] Hastings, W. K. (1970). Monte Carlo sampling methods using Markov chains and their applications. Biometrika 57 97-109.

[16] Johansen, A. M., Del Moral, P. and Doucet, A. (2006). Sequential Monte Carlo samplers for rare events. In Proceedings of the 6th International Workshop on Rare Event Estimation 256-267.

[17] KAhn, H. and Harris, T. E. (1951). Estimation of particle transmission by random sampling. National Bureau of Standards Appl. Math. Series 12 27-30.

[18] Lagnoux, A. (2006). Rare event simulation. Probability in the Engineering and Informational Sciences 20 45-66. MR2187629

[19] Le Gland, F. and Oudjane, N. (2006). A sequential algorithm that keeps the particle system alive. In Stochastic Hybrid Systems : Theory and Safety Critical Applications, (H. Blom and J. Lygeros, eds.). Lecture Notes in Control and Information Sciences 337 351-389. Springer-Verlag, Berlin.

[20] Merhav, N. and Sabbag, E. (2008). Optimal watermarking embedding and detection strategies under limited detection resources. IEEE Trans. on Inf. Theory $\mathbf{5 4}$ 255-274.

[21] Metropolis, N., Rosenbluth, A. W., Rosenbluth, M. N., Teller, A. H. and Teller, E. (1953). Equation of State Calculations by Fast Computing Machines. The Journal of Chemical Physics 21 1087-1092.

[22] Meyn, S. P. and Tweedie, R. L. (1993). Markov chains and stochastic stability. Communications and Control Engineering Series. Springer-Verlag London Ltd., London. MR1287609 (95j:60103)

[23] Rosenbluth, M. N. and Rosenbluth, A. W. (1955). Monte Carlo Calculation of the Average Extension of Molecular Chains. Journal of Chemical Physics 23.

[24] Rubinstein, R. (2008). The Gibbs Cloner for Combinatorial Optimization, Counting and Sampling. Methodology and Computing in Applied Probability.

[25] Tardos, G. (2003). Optimal probabilistic fingerprint codes. In Proc. of the 35th annual ACM symposium on theory of computing 116-125. ACM, San Diego.

[26] Tierney, L. (1994). Markov chains for exploring posterior distributions. Ann. Statist. 22 1701-1762. With discussion and a rejoinder by the author. MR1329166 (96m:60150)

[27] VAn Der VAart, A. W. (1998). Asymptotic statistics. Cambridge Series in Statistical and Probabilistic Mathematics. Cambridge University Press, Cambridge. MR1652247 (2000c:62003) 
inRia Rennes - Bretagne Atlantique, inRia Bordeaux Sud-Ouest \& Institut de Mathématiques de Bordeaux, Campus de Beaulieu, 35042 Rennes Cedex, France Université BordeaUX 1,

E-MAIL: frederic.cerou@irisa.fr

351 cours de la libération, 33405 Talence Cedex, France teddy.furon@inria.fr

E-MAIL: Pierre.Del_Moral@inria.fr

Université de Haute Bretagne,

Place du Recteur H. Le Moal, CS 24307,

35043 Rennes Cedex, France

E-MAIL: arnaud.guyader@uhb.fr 